\title{
Accumulated Clinical Experiences from Successful Treatment of 1377 Severe and Critically Ill COVID-19 Cases*
}

Yong $\mathrm{GAO}^{1 \dagger}$, Hai-bo QIU ${ }^{2 \dagger}$, Shu ZHOU ${ }^{1}$, Zhen-ning WANG ${ }^{3}$, Jian-chu ZHANG ${ }^{1}$, Zheng-liang ZHANG ${ }^{4}$, Zhao-xin QIAN ${ }^{5}$, Hong-bo WANG ${ }^{1}$, Shi-huan YU ${ }^{6}$, Yi-feng LUO ${ }^{7}$, Ya-dan WANG ${ }^{1}$, Zhuang LIU ${ }^{8}$, Wei-ci WANG ${ }^{1}$, Ming JIA ${ }^{9}$, Li-ming ZHANG ${ }^{10}$, Yu ZHANG ${ }^{1}$, Yang JIN ${ }^{1}$, Yong ZHANG ${ }^{1}$, Yong LIU ${ }^{1}$, Zi-hua ZHOU ${ }^{1}$, Ying SU ${ }^{1}$, Hui-qing LI ${ }^{1}$, Wei-min XIAO ${ }^{1}$, Kai HUANG ${ }^{1}$, Ping HE ${ }^{1}$, Gang LI $^{1}$, Zhao-hui FU ${ }^{1}$, Shi LIU ${ }^{1}$, Neng-xing LIN ${ }^{1}$, Fan-jun CHENG ${ }^{1 \#}$

${ }^{1}$ Union Hospital, Tongji Medical College, Huazhong University of Science and Technology, Wuhan 430022, China

${ }^{2}$ Zhongda Hospital of Southeast University, Nanjing 210009, China

${ }^{3}$ The First Affiliated Hospital of China Medical University, Shenyang 110001, China

${ }^{4}$ The Second Affiliated Hospital of Xi' an Jiaotong University, Xi'an 710004, China

${ }^{5}$ Xiangya Hospital of Zhongnan University, Changsha 410008, China

${ }^{6}$ The First Affiliated Hospital of Harbin Medical University, Harbin 150001, China

${ }^{7}$ The First Affiliated Hospital of Sun Yat-sen University, Guangzhou 510080, China

${ }^{8}$ Beijing Friendship Hospital of Capital Medical University, Beijing 100050, China

${ }^{9}$ Beijing Anzhen Hospital of Capital Medical University, Beijing 100029, China

${ }^{10}$ Beijing Chaoyang Hospital of Capital Medical University, Beijing 100020, China

(C) Huazhong University of Science and Technology 2020

\begin{abstract}
Summary: In late December 2019, COVID-19 was firstly recognized in Wuhan, China and spread rapidly to all of the provinces of China. The West Campus of Wuhan Union Hospital, the designated hospital to admit and treat the severe and critically ill COVID-19 cases, has treated a large number of such patients with great success and obtained lots of valuable experiences based on the Chinese guideline (V7.0). To standardize and share the treatment procedures of severe and critically ill cases, Wuhan Union Hospital has established a working group and formulated an operational recommendation, including the monitoring, early warning indicators, and several treatment principles for severe and critically ill cases. The treatment experiences may provide some constructive suggestions for treating the severe and critically ill COVID-19 cases all over the world. Key words: 2019-nCoV; COVID-19; pneumonia; treatment; guidelines
\end{abstract}

An ongoing outbreak of pneumonia caused by the 2019 novel coronavirus (2019-nCoV) has been recently recognized as a newly emerging infectious disease. 2019-nCov is highly contagious, and it has a significant morbidity and $2 \%-4 \%$ mortality rate ${ }^{[1,2]}$. 2019-nCoV disease (COVID-19) was first identified in Wuhan, China in late December 2019, and it subsequently spread rapidly to all of the provinces in China ${ }^{[1]}$. The 2019-nCoV belongs to a novel type of $\beta$ genus coronavirus that shares $79.5 \%$ sequence identity to severe acute respiratory syndrome-related coronaviruses (SARS-CoV) ${ }^{[3]}$. It mainly, but not exclusively, attacks the human respiratory system in

Yong GAO, E-mail: docgao@163.com; Hai-bo QIU, E-mail: Haiboq2000@163.com

†The authors contributed equally to this work.

\#Corresponding author, E-mail: chengfanjun001@sina.com

*The work was supported by COVID-19 Emergency Scientific Research Project of Science and Technology Department, Hubei Province (No. 2020FCA041). severe and critically ill cases ${ }^{[4]}$. Among the infected patients, about $14 \%$ were severe and $5 \%$ were critically ill $^{[1]}$ who were commonly accompanied by cellular immune deficiency, coagulation activation, cytokine storm, myocardia injury, hepatic injury, and kidney injury. According to minimally invasive autopsies, we learned that the lungs from COVID-19 patients manifest significant pathological lesions and the injury also involves damage to the heart, vessels, liver, kidney, and other organs $s^{[5]}$.

Chinese health institutions enacted immediate measures to control the disease, including isolation of suspected people, close monitoring of contacts, collection of epidemiological and clinical data, and development of diagnostic and treatment procedures. The West Campus of Wuhan Union Hospital, as one of the three designated hospitals appointed by the National Health Commission of China to admit and treat the severe and critically ill COVID-19 cases, has recruited the largest number of these patients. As of April 1, 2020, 1638 patients who were considered to be severe 
and critically ill COVID-19 have been treated, and 1377 of these patients were cured and discharged, 167 patients died, and 94 patients are currently undergoing treatment. Most of the patients have been cured and the spread has been well controlled, indicating the effectiveness of our management and treatment.

Currently, the pandemic in other countries is still serious, which has prompted international concern of the global public-health impact ${ }^{[6]}$. To standardize and share the treatment procedures, Wuhan Union Hospital has established a working group and formulated the following operational recommendations for treating severe and critically ill patients. The manuscript is based on the Chinese guideline (V7.0) ${ }^{[7]}$ and previous experiences accumulated from the isolation ward over the past two months.

\section{ESTABLISHMENT OF BASELINE DISEASE DATA ON ADMISSION}

\subsection{Disease History Collection}

Upon admission, all of the disease data from the patients should be accurately collected. This serves as a starting point for observation and disease evolution. The data collected include epidemiological history, clinical manifestations, as well as the collection, collation, and recording of diagnosis and treatment data in other medical institutions. The severe and critically ill patients should be diagnosed according to the guidelines of the National Health Commission of China (V7.0) ${ }^{[7]}$. All of the data should be collected during treatment, and a flowchart should be established to depict and predict disease evolution.

\subsection{Auxiliary Examinations}

On admission, patients are required to complete the following tests:

1) Complete blood count, lymphocyte subsets;

2) C-reactive protein (CRP), erythrocyte sedimentation rate (ESR), procalcitonin (PCT), and inflammatory cytokines (including IL-6, IL-10, and TNF- $\alpha$ );

3) Liver and kidney function and electrolytes;

4) Disseminated intravascular coagulation (DIC) screening (including coagulation function and D-dimer);

5) Blood gas analysis;

6) Chest X-ray or CT scan;

7) Others: troponin, cardiac enzymes, B-type natriuretic peptide, lactate dehydrogenase (LDH), creatine kinase, and myoglobin.

It is recommended that the severe patients should be monitored every three days, and critically ill patients should be monitored daily for the above examinations except for radiographic images. The evolution curve can be generated through a data management program. 1.3 Detection of Pathogens

1) 2019-nCoV detection: 2019-nCoV nucleic acid can be detected in nasopharyngeal swabs, sputum, lower respiratory tract secretions, blood, feces, and other specimens utilizing RT-PCR or NGS methods. The accuracy of RNA 2019-nCoV detection is as follows: alveolar lavage fluid > sputum >nasal swab $>$ throat swab. 2019-nCoV-specific IgM becomes detectable via serological tests at 3-5 days post-onset, and an IgG-positive test indicates previous infection and convalescence. The combined detection of viral RNA and serology in COVID-19 patients can improve the sensitivity and specificity of diagnosis.

2) Secondary bacterial and fungal pathogenic examination: Severe and critically ill patients with COVID-19 are susceptible to bacterial and fungal infections, and clinical microbiological test should be monitored.

3) Metagenomic sequencing (mNGS): mNGS has obvious advantages of comprehensive detection, high accuracy, high sensitivity, and fast identification over RT-PCR, which not only significantly improved the detection efficiency of 2019-nCoV, but also improved the detection efficiency of co-infection and secondary infection of severe and critically ill COVID-19 patients.

4) Sampling, transportation, and testing involving pathogenic inspections must meet biosafety requirements. 1.4 Comorbidities and Complications Monitoring

1) Most of the patients belonging to the severe and critically ill type of COVID-19 are relatively older and generally have a variety of comorbidities. These pathophysiological factors have a great impact on the diagnosis, treatment, and prognosis of COVID-19, which should be noted and monitored;

2) 2019-nCoV affects multiple organs other than the respiratory system, including cardiovascular system, gastrointestinal system, blood, immunity, etc. The appropriate examinations should be carried out when necessary;

3) No specific drugs have yet been produced. Although several types of clinically therapeutic drugs have been tried, the interactions between drugs and drug damage are not clear and should be taken into consideration. The clinical pharmacy-related tests are required when necessary.

\section{CLINICAL EARLY WARNING INDICATORS OF SEVERE AND CRITICALLY ILL CASES}

Previous experiences have revealed that some COVID-19 cases can progress and deteriorate from moderate or severe, or to severe or critically ill in a short period of time. The cases with the following indicators are likely to deteriorate to severe or critically ill status. Early warning and treatment will be of great importance to reduce mortality.

1) Age $\geq 65$ years old, CRP $\geq 20 \mathrm{mg} / \mathrm{L}$, lymphocyte count $\leq 800 / \mu \mathrm{L}$, eosinophil deficiency $(\leq 100 / \mu \mathrm{L})$, 
pulmonary consolidation involving the inner band, abnormal DIC screening, etc. may be independent prognostic factors which can be regarded as early warning indicators of deterioration ${ }^{[1,2,8]}$. For the patients with any three of the above indicators, the first-aid treatment should be carried out as soon as possible to reduce the risk of mortality.

2) Elevated neutrophils, normal or declined lymphocytes and with or without the eosinophils decline, in addition to indicating a bacterial infection, also predict a poor prognosis.

3) Patients with age $\geq 65$ years and accompanied by serious underlying diseases (including structural lung disease, coronary heart disease, critical hypertension, rheumatic immune disease, neoplastic disease, other infectious diseases, etc.), immunosuppressive treatment, organ transplantation, blood purification or chemotherapy are recommended to be treated as severe type.

4) Patients with age $\geq 75$ years and accompanied by any organ dysfunction or uncontrolled serious underlying diseases are recommended to be treated as critically ill type.

5) Patients who have been treated for more than a week but without achieving significant improvement should be paid more attention to, and clinical monitoring and examinations should be strengthened. In case of exacerbation, empirical proactive treatment should be immediately implemented according to the treatment guideline of severe patients.

\section{PRINCIPLES FOR TREATING THE SEVERE AND CRITICALLY ILL CASES}

\subsection{Basic Treatment}

1) Let patients rest in bed and strengthen nutritional support therapy;

2) Maintain water, electrolyte, and acid-base balance;

3) Energy mixture, ATP, or CoA can be used for anti-hypoxia treatment;

4) Underlying diseases should be treated;

5) Measures should be taken to prevent secondary infection;

6) Prevent and treat complications, including: cardiac injury, cardiogenic shock, myocarditis, venous thrombosis (VTE), DIC, ventilator-induced dysfunction, and multi-functional organ failure;

7) Treat the cytokine storm.

\subsection{Antiviral Therapy}

Numerous randomized controlled trials (RCTs) have been conducted to evaluate the efficacy of antiviral drugs towards 2019-nCoV. Lopinavir can inhibit the protease activity of coronavirus, which has been used to control the SARS and MERS outbreaks and was recommended by Chinese guidelines to treat COVID-19, but it was recently reported that no benefit was observed with lopinavir-ritonavir treatment beyond standard care in a randomized, controlled, open-label trial ${ }^{[9]}$. Chloroquine phosphate and hydroxychloroquine that are reported to be potential broad-spectrum antiviral drugs with immunomodulatory effects, have been recommended to treat COVID-19 (500 mg for 7 days for adults aged 18-65 with body weight over $50 \mathrm{~kg}$; $500 \mathrm{mg}$ for days 1 and 2, and $500 \mathrm{mg}$ for days 3-7 for adults with body weight below $50 \mathrm{~kg}$ ), but the evidence on the benefits and harms is very weak ${ }^{[10]}$; and recently FDA has revoked emergency use authorization for chloroquine and hydroxychloroquine. Arbidol, a broad-spectrum antiviral drug, has also been under several clinical trials to evaluate the treatment efficacy towards COVID-19 in combination with lopinavir/ ritonavir, chloroquine phosphate, etc. ${ }^{[11,12]}$. Remdesivir belongs to the adenine nucleotide analogue antiviral drug, and has exhibited in vitro activity against Ebola virus and 2019-nCoV. However, the in-vivo killing ability toward 2019-nCoV in Remdesivir Clinical Trials was conflicting without statistically significant clinical benefits ${ }^{[13,14]}$. Since there were no specific anti2019-nCoV drugs so far, the treatment of COVID-19 was still under clinical trials; and we need to pay more attention to adverse reactions, contraindications and interactions of the above-mentioned drugs. If an intolerable toxic side effect occurs, the respective drug should be discontinued.

\subsection{Oxygen Therapy and Respiratory Support}

\subsubsection{High-flow Nasal-catheter Oxygenation (HFNC)}

HFNC is suitable for patients with hypoxemia and oxygenation index $\left(\mathrm{PaO}_{2} / \mathrm{FiO}_{2}\right)$ in $200-300 \mathrm{mmHg}$. During the implementation of HFNC treatment, the symptoms and signs of the patients should be closely monitored and evaluated every $20-30 \mathrm{~min}$. The following conditions indicate failure of HFNC treatment, and alternative respiratory support therapy should be added: (1) Hypoxemia cannot be improved $\left(\mathrm{SPO}_{2}<93 \%\right.$ ); (2) Respiratory frequency ( $\mathrm{RR} \geq 35$ beats/min); (3) Significantly hard respiration.

3.3.2 Non-invasive Ventilator (NIV) NIV is suitable for patients with hypoxemia and $\mathrm{PaO}_{2} / \mathrm{FiO}_{2}$ in $150-200$ $\mathrm{mmHg}$. During the implementation of NIV treatment, the symptoms and signs of the patients should be closely monitored and evaluated every $20-30 \mathrm{~min}$. The following situations indicate failure of NIV treatment, and alternative respiratory support therapy should be added in time: (1) Hypoxemia cannot be improved $\left(\mathrm{SPO}_{2}<93 \%\right)$; (2) $\mathrm{RR} \geq 35$ beats/min; (3) Excessive tidal volume; (4) Excessive negative inspiratory pressure; (5) Unstable circulation and abnormal tissue perfusion.

Invasive mechanical ventilation (IMV) is suitable for patients with hypoxemia and $\mathrm{PaO}_{2} / \mathrm{FiO}_{2}$ $<150 \mathrm{mmHg}$. Based on lung protective ventilation strategy ${ }^{[15]}$, the IMV treatment requires repeated monitoring of the lung recruitment maneuver potential. 
The medical staff should use the PEEP strategy to restore the lung potential, and closely observe the oxygenation index, pressure of carbon dioxide, right heart enlargement, and pulmonary barotrauma. Prone position ventilation is recommended. Patients who have reached the following conditions can withdraw mechanical ventilation: $\mathrm{PaO}_{2} / \mathrm{FiO}_{2}$ maintains $>200$ $\mathrm{mmHg}$, the primary disease is improved, the patient is conscious, and the patient's circulation is stable.

3.3.3 Extracorporeal Membrane Oxygenation (ECMO) Indications of ECMO therapy are as follows: $\mathrm{PaO}_{2} / \mathrm{FiO}_{2}<50 \mathrm{mmHg}$ for more than $3 \mathrm{~h} ;$ (2) $\mathrm{PaO}_{2} / \mathrm{FiO}_{2}$ $<80 \mathrm{mmHg}$ for more than $6 \mathrm{~h}$; (3) $\mathrm{FiO}_{2} 1.0, \mathrm{PaO}_{2} / \mathrm{FiO}_{2}$ $<100$ mmHg; (4) Arterial blood $\mathrm{pH}<7.25$ and $\mathrm{PaCO}_{2}$ $>60 \mathrm{mmHg}$ more than $6 \mathrm{~h}$, or related complications caused by carbon dioxide retention: severe internal environment disorder and right heart failure; (5)When the respiratory rate $>35$ per min, the arterial blood $\mathrm{pH}$ $<7.2$ and the airway platform pressure $>30 \mathrm{cmH}_{2} \mathrm{O}$; (6) Combined with cardiogenic shock or cardiac arrest.

The contraindications of ECMO therapy are as follows: Combined with irrecoverable primary diseases, anticoagulant contraindications, ventilation for more than 7 days under a higher mechanical ventilation setting $\left(\mathrm{FiO}_{2}>0.9\right.$, airway platform pressure $>30 \mathrm{cmH}_{2} \mathrm{O}$ ), age $>70$ years old, the use of immunosuppression, and the presence of peripheral vascular anatomical malformations or vascular lesions.

\subsection{Rational Use of Antibiotics}

1) Avoid blind or inappropriate use of antibiotics if there is no clear evidence of bacterial infection;

2) For severe and critical cases with a course of disease $\geq 7$ days, surveillance of pathogens related to secondary bacterial or fungal infection should be effectively carried out;

3) Bacterial infection should be suspected and confirmed according to body temperature, white blood cell count (WBC), neutrophil percentage, pulmonary imaging, oxygenation function, and pathogen examination. The third-generation cephalosporin/enzyme inhibitor complex can be empirically used;

4) When septic shock occurs in severe and critically ill patients, carbapenem drugs can be used instead. If enterococcal and staphylococcal infections are considered, glycopeptide drugs (vancomycin) can be added for empirical treatment;

5) Enhanced attention should be paid to catheterrelated infections. Empirical antibiotic treatment should cover methicillin-resistant staphylococci with the use of glycopeptide drugs (vancomycin);

6) Some patients often have secondary aspergillosis infection in the later stages of critical illness. Voriconazole can be used, but the combination of two antifungal drugs is not recommended;

\subsection{Prophylactic Anticoagulant Therapy}

1) Severe and critically ill COVID-19 patients have a higher risk of DIC and VTE. Coagulation and bleeding should be closely monitored during treatment. Unless there is significant bleeding or a coagulation disease, low-molecular-weight heparin is recommended for the vast majority of critically ill patients.

2) When DIC occurs and there is no significant hyperfibrinolysis, a therapeutic dose of low-molecularweight heparin can be added, but simultaneous replacement therapy, including platelet transfusion, and/or fresh plasma should be supplemented to replenish coagulation factors; hematologists should be invited to consult as soon as possible;

3) The risk of VTE in severe and critically ill patients should be regularly assessed according to the Caprini Risk Assessment Model: when the score $>3$, drug prevention and physical prevention are recommended. Encourage patients to exercise at an early stage and invite a vascular surgeon to consult when VTE is suspected.

\subsection{Convalescent Plasma Treatment}

The 2019-nCoV-specific antibodies in the plasma of COVID-19 convalescent patients have a certain therapeutic effect on COVID-19 patients via reducing 2019-nCoV content. Convalescent plasma treatment can be used for severe patients $>18$ years of age, and only for the certain critical patients with mechanical ventilation $\leq 48 \mathrm{~h}$ and heart, liver, and kidney functions still being in a compensatory state.

\subsection{IL-6 Monoclonal Antibody Treatment}

Cytokine release syndrome (CRS), which is closely associated with the increased level of IL-6, is an important cause of death in critically ill patients with COVID-19. It is speculated that the IL-6 monoclonal antibody (Tocilizumab) can inhibit the intensity of the cytokine storm and play a certain therapeutic role. IL-6 monoclonal antibody therapy is suitable for severe patients $>18$ years of age without severe comorbidities, and forbidden for patients with active infections, such as tuberculosis.

\subsection{Glucocorticoids Treatment}

Recently, there are reports supporting that dexamethasone can reduce death rate of the hospitalized patients with severe respiratory complications of COVID-19 ${ }^{[16,17]}$. Patients with progressive deterioration of oxygenation indicators, rapid progress in imaging, excessive activation of the body's inflammatory response, and without contraindications can use conventional doses of glucocorticoids in a short period of time (3-5 days).

\subsection{Other Recommended Treatments}

There is no medical evidence to support the application of drugs such as human immunoglobulin, thymosin, and intestinal microecological regulators to improve the prognosis of severe COVID-19 patients. They are recommended as supplemental therapeutic 
measures.

\subsection{Traditional Chinese Medicine}

COVID-19 belongs to the category of traditional Chinese medicine plagues. Dialectical treatment based on factors, such as seasonal and geographical aspects, has played an important role in the prevention and control of the COVID-19 epidemic in China from 2019 to 2020. According to the different local climate characteristic and individual state of illness and physical conditions, Chinese medicine prescriptions and Western medicines can be used alone or in combination.

\section{Acknowledgements}

We appreciate all of the doctors and nurses who share their valuable experiences and for their hard work in treating COVID-19 patients, and for those who have contributed to translating and proofreading the manuscript.

\section{Conflict of Interest Statement}

The authors declare that they have no competing interests.

\section{REFERENCES}

1 Wu Z, Mcgoogan JM. Characteristics of and Important Lessons From the Coronavirus Disease 2019 (COVID-19) Outbreak in China: Summary of a Report of 72314 Cases From the Chinese Center for Disease Control and Prevention. JAMA, 2020

2 Wang DW, Hu B, Hu C, et al. Clinical Characteristics of 138 Hospitalized Patients With 2019 Novel Coronavirus-Infected Pneumonia in Wuhan, China. JAMA, 2020,323(11):1061-1069

3 Zhou P, Yang X, Wang X, et al. A pneumonia outbreak associated with a new coronavirus of probable bat origin. Nature, 2020,579(7798):270-273

4 Huang C, Wang Y, Li X, et al. Clinical features of patients infected with 2019 novel coronavirus in Wuhan, China. Lancet, 2020,395(10223):497-506

5 Yao XH, Li TY, He ZC, et al. A pathological report of three COVID-19 cases by minimally invasive autopsies. Zhonghua Bing Li Xue Za Zhi (Chinese), 2020,49(0):E009

6 Wang C, Horby PW, Hayden FG, et al. A novel coronavirus outbreak of global health concern. Lancet, 2020,395(10223):470-473
7 National Health Commission. The guidelines for the diagnosis and treatment of 2019 novel coronavirus (2019-nCoV) infection (Pilot 7rd version). [S/OL]. 2020(2020-03-03).

8 Chen N, Zhou M, Dong X, et al. Epidemiological and clinical characteristics of 99 cases of 2019 novel coronavirus pneumonia in Wuhan, China: a descriptive study. Lancet, 2020,395(10223):507-513

9 Cao B, Wang Y, Wen D, et al. A Trial of LopinavirRitonavir in Adults Hospitalized with Severe Covid-19. N Engl J Med, 2020,382(19):1787-1799

10 Hernandez AV, Roman YM, Pasupuleti V, et al. Hydroxychloroquine or Chloroquine for Treatment or Prophylaxis of COVID-19: A Living Systematic Review. Ann Int Med, 2020.

11 Deng L, Li C, Zeng Q, et al. Arbidol combined with LPV/r versus LPV/r alone against Corona Virus Disease 2019: A retrospective cohort study. J Infect, 2020,81(1):e1-e5

$12 \mathrm{Zhu} \mathrm{Z}, \mathrm{Lu} \mathrm{Z}, \mathrm{Xu} \mathrm{T}$, et al. Arbidol monotherapy is superior to lopinavir/ritonavir in treating COVID-19. J Infect, 2020,81(1):e21-e23

13 Beigel JH, Tomashek KM, Dodd LE, et al. Remdesivir for the Treatment of Covid-19 - Preliminary Report. N Engl J Med, 2020:NEJMoa2007764

14 Grein J, Ohmagari N, Shin D, et al. Compassionate Use of Remdesivir for Patients with Severe Covid-19. N Engl J Med, 2020,382(24):2327-2336

15 Fan E, Del Sorbo L, Goligher EC, et al. An Official American Thoracic Society/European Society of Intensive Care Medicine/Society of Critical Care Medicine Clinical Practice Guideline: Mechanical Ventilation in Adult Patients with Acute Respiratory Distress Syndrome. Am J Respir Crit Care Med, 2017,195(9):1253-1263

16 Ledford H. Coronavirus breakthrough: dexamethasone is first drug shown to save lives [published online ahead of print, 2020 Jun 16]. Nature, 2020:10.1038/d41586020-01824-5

17 Abdolahi N, Kaheh E, Golsha R, et al. Letter to the editor: efficacy of different methods of combination regimen administrations including dexamethasone, intravenous immunoglobulin, and interferon-beta to treat critically ill COVID-19 patients: a structured summary of a study protocol for a randomized controlled trial. Trials, 2020,21(1):549

(Received May 5, 2020; accepted June 30, 2020) 